\title{
Cerebral computed tomographic angiography scan delay in subarachnoid hemorrhage
}

\author{
Saulius Lukosevicius, Algidas Basevicius, Arimantas Tamasauskas* \\ Departments of Radiology and *Neurosurgery, Kaunas University of Medicine, Lithuania
}

\begin{abstract}
Context: Computed tomographic angiography (CTA) is widely applied in the evaluation of cerebral vessels. Contrast enhancement in cerebral CTA without care or test bolus is not always sufficient for high-quality images. Aims: Evaluation of the possibilities of calculation of scan delay for cerebral CTA in case of subarachnoid hemorrhage (SAH), based on clinical data of a patient and to find out prognostic error of the model. Settings and Design: Prospective study in Neurosurgery and Radiology departments. Materials and Methods: Scan delay in 53 patients suffering an acute SAH was measured employing test bolus technique. Cerebral CTA was performed afterwards. Statistical Analysis Used: SPSS for Windows v.10.1 software package was applied for dispersion analysis, including one-sample KolmogorovSmirnov's test and Levene's Test of Equality of Error Variances. Results: A statistical model for the prediction of scan delay in SAH was developed. Cerebral CTA scan delay was dependent upon age, neurological status and impact of the latter factors together $(P<0.05)$. The determined mean square error of prognosis of scan delay of the developed model equals $3.3 \mathrm{sec}$. Conclusion: Using our proposed model it is possible to estimate an optimal delay time for CTA in most patients with SAH with a determined error.
\end{abstract}

Key Words: Cerebral computed tomographic angiography, scan delay, subarachnoid hemorrhage

\section{Introduction}

Morphological alterations of brain vessels, like cerebral aneurysms, arteriovenous malformations (AVM) or stenosis of cerebral arteries are best depicted by means of digital subtraction angiography (DSA). It is still considered as a "gold standard" in this sphere, despite its invasiveness and potential for complications. ${ }^{[1-3]}$ In the last decades of the $20^{\text {th }}$ century the newly developed minimally invasive angiography meth- ods based on spiral computed tomography (CT) and magnetic resonance (MR), i.e. computed tomographic angiography (CTA) and magnetic resonance angiography (MRA) appeared in the field of diagnosis of cerebrovascular diseases.

In 1987 brain vasculature was investigated with non-spiral CT for the first time. ${ }^{[4]}$ However, most of the publications deal with the application of CTA in case of presence of cerebral aneurysms and subarachnoid hemorrhage (SAH) ${ }^{[5-10]}$ and also in case of ischemic stroke ${ }^{[11,12]}$ and AVM. ${ }^{[13-15]}$ In some publications there is a trend of increasing number of neurosurgical operations based solely on CTA data, without initial preoperative DSA, thus indicating a tremendous increase in accuracy, specificity and sensitivity, as well as of trust in this method among operating neurosurgeons. ${ }^{[7]}$

Various complex interrelationships affect the use of contrast media for CTA, thus the technique for delivery of contrast medium remains one of the main controversial aspects of CTA. ${ }^{[16-19]}$ An important point regarding the efficient quality of any kind of CTA examination is adequate and time-related arterial enhancement. ${ }^{[8,20]}$ Previous studies incorporate the evaluation of the influence of technique-related ${ }^{[16,20,21]}$ and patient-related factors ${ }^{[17,21-23]}$ onto scan delay with no attempts at finding out important clinical factors and using them in the determination of scan delay before CTA examination. Our study attempts to find out the predictive clinical factor as well as develop a statistical model suitable to calculate with a definite error the exact time delay in case of SAH, based on certain clinical factors, without additional pre-scan (i.e. test bolus).

\section{Materials and Methods}

\section{Patients}

The study was a constituent part of a larger scale study on cerebral CTA in case of SAH that was performed in the Departments of Neurosurgery and Radiology, during 1998 to 2002. During the period of study (1998-2002) 445 patients were admitted to University 
Hospital because of SAH, which was confirmed by patient history and plain CT and/or by lumbar puncture. Inclusion criteria: SAH on plain CT or confirmed by lumbar puncture, no signs of intracerebral hemorrhage (ICH), delay between the onset of symptoms of SAH and cerebral CTA performance - not more than 3 days, performance of test bolus procedure prior to cerebral CTA. CTA examinations included in this study were only day-time examinations because of the limited presence of experienced radiologists and radiology-assistants round the clock. Exclusion criteria: known adverse reaction to contrast media, pregnancy, renal failure, refusal to undergo the procedure.

Fifty-three patients with SAH underwent cerebral CTA with test bolus procedure. The median age of the patients was 51,8 $\pm 13,2$ years (17 to 86 years). Two patients were referred to age group 1 ( $<30 \mathrm{y})$, 40 patients to age group $2(30-60 \mathrm{y})$ and 11 patients to age group 3 (>60 y). 41,5\% (22/53) were male patients, $58,5 \%$ (31/53) were females. The condition of patients was graded according to the Hunt \& Hess (H\&H) scale. ${ }^{[24]}$ Four patients were in H\&H Grade I, 32 patients in H\&H Grade II, 12 patients in H\&H Grade III, and 5 in H\&H Grade IV. The extent of SAH on CT was graded according to the Fisher grading scale. ${ }^{[25]}$ Fifty patients had SAH on plain CT (17 referred to Fisher Grade 2, 16 to Fisher Grade 3, 17 to Fisher Grade 4). Three patients had SAH confirmed by lumbar puncture in regional hospitals (Fisher Grade 1, n=3) (Table 1).

All the procedures were carried out in accordance with the ethical standards of the responsible committee of the Kaunas University of Medicine and with the Helsinki Declaration of 1975, revised in 2000.

\section{Equipment}

Scanning was performed with the help of spiral CT unit Siemens Somatom Plus 4 (Siemens AG), whilst multiphase injector (OP 100, CT Multilevel CT injector, Medrad) was employed to inject $100 \mathrm{ml}$ of non-ionic contrast medium (300 mg I/ml) via $18-20$ G standard intravenous catheter into the antecubital vein of a patient. In case of test bolus $-17-18 \mathrm{ml}$ of contrast medium were injected for test bolus and the rest $82-83 \mathrm{ml}$ were injected in the second phase of cerebral CTA.

\section{Methods}

All 53 patients underwent a test bolus procedure prior to cerebral CTA (Figures 1, 2). The exact peak time for enhancement was recorded for every investigated patient at the level of the cavernous section of the internal carotid artery, or elsewhere in the anterior

Table 1: Number and clinical factors of patients with SAH, who underwent test bolus procedure prior to cerebral CTA

\begin{tabular}{lcc}
\hline & $\begin{array}{c}\text { Groups, signs, } \\
\text { symptoms, etc. }\end{array}$ & $\begin{array}{c}\text { Number of } \\
\text { patients }\end{array}$ \\
Neurological status & I grade & 4 \\
(Hunt \& Hess grade) & II grade & 32 \\
& III grade & 12 \\
IV grade & 5 \\
Age & $<30$ y & 2 \\
& $30-60 y$ & 40 \\
Gender & $>60 y$ & 11 \\
SAH extent on CT & Males & 22 \\
(Fisher grade) & Females & 31 \\
& 1 & 3 \\
& 2 & 17 \\
& 3 & 16 \\
& 4 & 17 \\
\hline
\end{tabular}

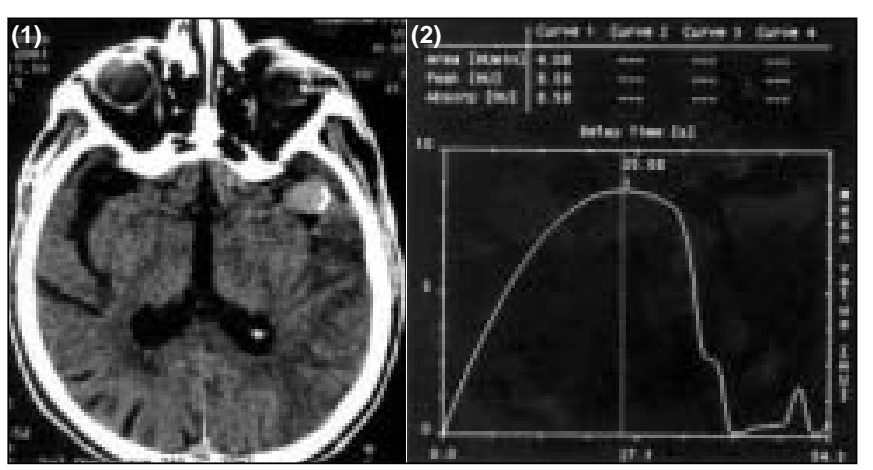

Figure 1: Region of interest in the anterior circulation, including the suspected aneurysm. Figure 2: Time-enhancement curve in the region of interest

circulation: in case of aneurysm suspicion, in the suspected region, otherwise in the cavernous section of the internal carotid artery itself. The scan delay time was the time to peak of enhancement on the time-attenuation curve obtained with the test injection. ${ }^{[16]}$ The following technical parameters were applied for cerebral CTA test bolus procedure for investigation of circle of Willis: Slice $5 \mathrm{~mm}$ (140 $\mathrm{kV}$ and $223 \mathrm{mAs}$ ); Rotation time $1 \mathrm{~s}$; Delay of the first scan $10 \mathrm{~s}$; Scan plane at the level of the cavernous sinus or location of the suspected aneurysm of anterior circulation; Number of scans 13 (each $2 \mathrm{~s}$ ); Injection flow speed $2.8 \mathrm{ml} / \mathrm{s}$ (same like cerebral CTA); Injection volume 17-18 ml. Technical parameters applied for the investigation of circle of Willis were Collimation $1 \mathrm{~mm}(120 \mathrm{kV}$ and 130 $\mathrm{mA}$ ); Pitch 1.5; Rotation time $1 \mathrm{~s}$; Increment 0.8 ; No gantry tilt; Direction caudo-cranial; Injection flow speed $2.8 \mathrm{ml} / \mathrm{s}$; Injection volume up to $100 \mathrm{ml}$; Scan delay detected by test bolus for each patient. Results of cerebral CTA will not be referred to or discussed in this paper:

Dispersion analysis was performed in order to evaluate the influence of certain clinical factors (Table 1). The possibility of the application of dispersion analysis was checked by means of the evaluation of normal distribution of å component of the model (component which reflects the influence of all unknown impact factors), and the evaluation of the hypothesis of equality of dispersion variances was also performed.

\section{Results}

The patients' characteristics are presented in Table 1 . The impact of age and neurological status on scan delay times is presented in Tables 2, 3 .

After evaluation of the results it is evident that only neurological status and age have a significant effect on scan delay, according to our data $(P<0.05)$. We also predict that one more factor - the co-influence of age and neurological status together also may alter time delay for cerebral CTA. Thus a proposed modified statistical model must incorporate intercept, influence of age, neurological status, as well as co-influence of age and neurological status together. Table 4 demonstrates that time delay depends upon the intercept $(\mu)$, age, neurological status and the combination of the latter. The influence of $\varepsilon$ member is also encountered in the model. These factors commit up to $78 \%$ of all detected time delay dispersion (as $\mathrm{R}$ squared is 0.78 ). 
Table 2: Mean of scan delay with respect to age group

\begin{tabular}{lcc}
\hline Age group & Mean & Std. Deviation \\
$<30$ yrs. & 15.50 & 0.71 \\
$30-60$ yrs. & 16.85 & 6.01 \\
$>60$ yrs. & 29.45 & 5.05 \\
\hline
\end{tabular}

\begin{tabular}{lcc}
\hline \multicolumn{3}{l}{ Table 3: Mean of scan delay with respect to neurological status } \\
\hline $\begin{array}{l}\text { Neurological status } \\
\text { (Hunt and Hess grade) }\end{array}$ & Mean & Std. Deviation \\
1. & 16.00 & 4.24 \\
2. & 15.41 & 4.76 \\
3. & 25.08 & 4.62 \\
4. & 34.20 & 2.95 \\
\hline
\end{tabular}

Table 4: Influence of significant factors on scan delay (dependent variable - scan delay)

\begin{tabular}{lcccc}
\hline Source & $\begin{array}{c}\text { Type III Sum } \\
\text { of Squares } \\
2475.5^{\mathrm{a}}\end{array}$ & $\mathbf{d f}$ & Mean Square & Sig. \\
Corrected model & 10636.4 & 1 & 353.6 & $<0.05$ \\
$\mu$ & 267.9 & 2 & 10636.4 & $<0.05$ \\
Age & 516.6 & 3 & 172.9 & $<0.05$ \\
$\begin{array}{l}\text { Neurological status } \\
\text { (Hunt and Hess grade) }\end{array}$ & & & & $<0.05$ \\
$\begin{array}{l}\text { Neurological status } \\
\text { and age together }\end{array}$ & 171 & 2 & 85.5 & $<0.05$ \\
$\begin{array}{l}\text { Error } \\
\text { Total }\end{array}$ & 591.4 & 45 & 13.1 & \\
\hline
\end{tabular}

${ }^{\mathrm{a}} \mathrm{R}$ Squared $=0.81$ (Adjusted R Squared 0.78).

Here: $\mathrm{df}-$ degrees of freedom; Mean square $=$ Sum of Squares $/ \mathrm{df} ; \mathrm{F}-$ Fisher's coefficient ( $\mathrm{F}=$ Mean Square / Error); Sig. - significance level; $\mu-$ intercept of the developed model.

As the influence of more than two impact factors is significant we propose the following table. Table 5 details the investigated parameters: neurological condition (Hunt \& Hess grade), age groups and influence of the latter factors together, as well as their statistical significance. The last line of each parameter in each group is set to zero, i.e. it's impact on scan delay is set to maximum, compared to other lines. The parameter estimates allow to evaluate the influence of all significant factors and calculate the individual scan delay using our proposed model.

The application of the model is rather simple. For example, scan delay for a patient in Hunt \& Hess Grade II, and age group 2 (30-60 y) will be the sum of all significant components: (Intercept) + (influence of neurological condition) + (influence of age) + (influence of neurological condition and age together $)=35.3+(-6.3)+(-2.8)+(-11.7)=14,5$ sec.

The influence of Hunt \& Hess Grade I (in Table 4 it appears as "[H\&H=1]") is "-16.5", i.e. the influence of this neurological condition on the scan delay is negative in relation to intercept (compared to the influence of Hunt \& Hess Grade $\mathrm{IV}$, which is set to zero, i.e. set to maximum).

While analyzing the influence of age on the time delay it is evident that the $1^{\text {st }}$ age group ( <30 y) affects time delay negatively - “-13.5". Evaluation of the $2^{\text {nd }}$ age group is not statistically significant due to the wide confidence interval and wide scatter $(P \geq 0.05)$. Scan delay is influenced significantly by the factor of the $1^{\text {st }}$ age group $(P<0.05)$, while there is no significant impact of the $2^{\text {nd }}$ age group $(P=0.39)$.

Also, it is evident that for patients in a poor neurological condition scan delay should be longer - significant impact on the scan delay in case of Hunt \& Hess Grade I and III (and with some compromise - Hunt \& Hess Grade II), confidence interval being $90 \%$ (Table 5 ).

Summarizing the calculations (Figure 3), we can conclude that in case of SAH, scan delay appears longer in elder patients, as well as in patients with more severe neurological status (Hunt \& Hess grade). It is especially evident in patients above 60 years of age and with Hunt \& Hess Grade IIIIV.

Based on our results it is possible to determine the mean square error of prognosis of time delay, calculated with the model:

$$
\Delta=\sqrt{\frac{1}{n} \sum_{i=1}^{n}\left(T_{\text {mox }}-T_{\text {nail }}\right)} ;
$$

Here: $\Delta$ - prognostic error; $\mathrm{n}$ - number of patients; $\mathrm{T}_{\text {prog }}-$ prognostic value of scan delay; $\mathrm{T}_{\text {real }}$ - real measured scan delay, applying test bolus procedure. According to our obtained results the prognostic error of our model $\Delta=3.3 \mathrm{sec}$.

\section{Discussion}

As mentioned above, this study was a constituent part of a larger scale study on cerebral CTA in case of SAH, that was performed in the Kaunas Medical University Hospital; however, the results of cerebral CTA and all sorts of comparison with DSA are beyond the purview of this paper. After some cases of cerebral CTA with missed peak of enhancement, indistinct cerebral vessels and insufficient quality of images, and also shortage of experienced radiological assistants round the clock, we decided to try and find out if it was possible to predict in a certain way (other than test bolus techniques), with a definite error, the exact time delay in case of SAH. It is important to stress that there is no possibility of installing care bolus upgrading into our CT machine.

It is evident that various factors related to the condition of a patient as well as the injection technique affect the use of contrast media for CTA. ${ }^{[16-19]}$ Starting from a paper published by Schad et al $^{[26]}$ in 1981, studies analyzing the technical problems of CTA including time delay could be divided into two major groups: dealing with technical ${ }^{[16,20,21]}$ and clinical ${ }^{[17,22,23]}$ factors. After the evaluation of the impact of heart rate, blood pressure, body length, weight, body surface area, or cardiac function Hartmann et $\mathrm{al}^{[17]}$ concluded that the prediction of individual scan delay from easily obtainable clinical parameters in case of acute pulmonary embolism was not possible. Although the authors pointed out that contrast transit times 
Table 5: Parameter estimates of the model (dependent variable - scan delay)

Intercept $(\mu)$

Influence of neurological condition

Influence of age

Influence of neurological condition and age together

\section{Parameters}

35.3

$[\mathrm{H} \& \mathrm{H}=1]$

$[\mathrm{H} \& \mathrm{H}=2]$

$[\mathrm{H} \& \mathrm{H}=3]$

$[\mathrm{H} \& \mathrm{H}=4]$

[age_gr=1]

[age_gr=2]

[age_gr=3]

$[\mathrm{H} \& \mathrm{H}=1]{ }^{*}$ [age_gr=2]

$[\mathrm{H} \& \mathrm{H}=2]$ * [age_gr=1]

$[\mathrm{H} \& \mathrm{H}=2]$ * [age_gr=2]

$[\mathrm{H} \& \mathrm{H}=2]$ * [age_gr=3]

$[\mathrm{H} \& \mathrm{H}=3]$ * [age_gr=2]

$[\mathrm{H} \& \mathrm{H}=3]$ * [age_gr=3]

$[\mathrm{H} \& \mathrm{H}=4]$ * [age_gr=2]

$[\mathrm{H} \& \mathrm{H}=4]$ * [age_gr=3]

$\begin{array}{cc}\text { B } & \text { SE } \\ 2.1 & <0 \\ -16.5 & 3.1 \\ -6.3 & 3.3 \\ -8.7 & 2.6 \\ 0^{\mathrm{a}} & \\ -13.5 & \\ -2.8 & 3.6 \\ 0^{\mathrm{a}} & \\ 0^{\mathrm{a}} & \\ 0^{\mathrm{a}} & \\ -11.7 & \\ 0^{\mathrm{a}} & \\ -0.3 & \\ 0^{\mathrm{a}} & \\ 0^{\mathrm{a}} & \\ 0^{\mathrm{a}} & \end{array}$

\section{SE}

$<0.05$

3.1

3.3

2.6

3.6

3.3

4.2

3.9

.

.
Sig.

$<0.05$

0.06

$<0.05$

$<0.05$

0.39

.

$<0.05$

0.93

aThis parameter is set to zero because it is redundant. Confidence interval- $90 \%$.

Here: B - model coefficient; SE - standard error of coefficient B; [H\&H=1] - neurological condition (corresponding Hunt \& Hess Grade); [age_gr=1] - corresponding age group, where age group $1-<30 \mathrm{y}$, age group $2-30-60 \mathrm{y}$, age group $3->60 \mathrm{y} ;[\mathrm{H} \& \mathrm{H}=1]$ * [age_gr=2] - combination of neurological condition $(\mathrm{Hunt} \&$ Hess grade) and of corresponding age group.

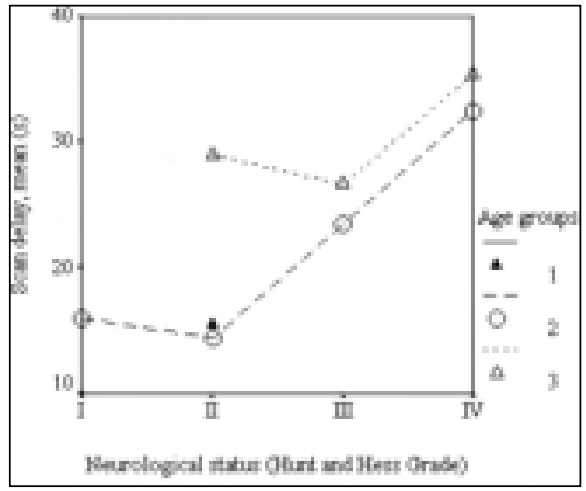

Figure 3: Relationship between scan delay, neurological status and age of the patients with SAH. Here: Age group 1- $<30$ y, age group $2-30-60$ $y$, age group $3->60$ y

were significantly related to gender and age, only $14.8 \%$ of the variation could be explained by these clinical parameters. Van Hoe et al ${ }^{[21]}$ pointed out a very weak correlation between patient age and peak enhancement level in abdominal CTA and detected no correlation with patient weight and heart rate. Puskas et $\mathrm{al}^{[23]}$ stated that there was no correlation of individual time delay with systolic or diastolic blood pressure, heart rate or body length, body weight or body surface in cerebral CTA, though the ROI in this study was placed on cerebral base arteries contralateral to the pathology. Nakajima et al ${ }^{[20]}$ confirmed that time to maximum contrast concentration was affected by patient age, WFNS grade and the post-resuscitation state from cardiopulmonary arrest. These findings are very similar to those in the present report. Yet the authors pointed out the necessity of performing dynamic prescan which is sometimes expensive and time-consuming. Our study suggests that scan delay individualization in patients with acute $\mathrm{SAH}$ is possible by means of a statistical model without any additional prescan. Hittmair and Fleischmann ${ }^{[19]}$ demon- strated that even injection site is important - arterial enhancement in CTA can be mathematically predicted and controlled more accurately if a central venous injection site is used. We did not try to find out any new or evaluate proven impact factors (e.g. metabolic status, hydration, medication, hypertension, medication, duration from symptoms of SAH to imaging, etc.) as it is not possible to guess all of them and this could be regarded as a limitation of the study. To solve this problem we added to our model a figure å, which represents the influence of all unknown factors on scan delay time.

According to our data, time delay for cerebral CTA in case of acute SAH depends upon neurological status (Hunt \& Hess grade), patient's age, as well as the combination of the two $(P<0.05)$. In consensus with other authors ${ }^{[22]}$ and based on our results we conclude that scan delay depends neither upon gender, nor SAH extent of CT images (Fisher grade) $(P>0.05)$. Thus scan delay must be prolonged in case of an older patient or in one with a more severe neurological condition. The mean square error of the prognosis of the model (prognostic error) is $3.3 \mathrm{sec}$.

Individual time delay could be detected with the help of statistical models - this could save time and eliminate additional contrast medium injection, as well as lower ionizing radiation dose, and probably reduce expenditures.

Of course, the study has certain limitations. One of them is the proven limitation of peripheral injection as compared to central injection of contrast media. ${ }^{[19]}$ There is no comparison of the influence of other grading scales of a patient's neurological condition (e.g., WFNS or Glasgow Coma Scale, etc.) on the duration of time delay, and there is also no comparison of scan delay in the anterior and posterior circulation basin. The influence of Hunt \& Hess Grade II on time delay is not statistically significant in case of $95 \%$ confidence interval, so confidence interval was lowered to $90 \%$ on purpose. Also the 
evaluation of the $2^{\text {nd }}$ largest age group is not statistically significant due to wide confidence interval and scatter $(P>0.05)$. Despite these and other possible imperfections of the study we presume that it is possible to predict time delay for cerebral CTA in case of SAH, based on certain clinical parameters, and it is obvious that not all of the influencing parameters are fully revealed. Further studies are required to evaluate the benefit of statistical models applied for scan delay determination in CTA.

\section{Conclusion}

Using our proposed table it is possible to estimate an optimal delay time for CTA in most patients with SAH with a determined error.

\section{Acknowledgement}

We thank Dr. Leonards Vilkauskas, recently retired Professor of the Department of Mathematies and Statisties of Kaunas Vytautas Magnus University, Lithuania for help with the statisties.

\section{References}

1. Berteloot D, Leclere X, Leys D, Krivosic R, Pruvo JP. Cerebral angiography: A study of complications in 450 consecutive procedures. J Radiol 1999;80:843-8

2. Cloft HJ, Joseph GJ, Dion JE. Meta-analysis of risks of cerebral angiography in patients with subarachnoid hemorrhage, intracranial aneurysm and arteriovenous malformation. Stroke 1999;30:317-20.

3. Leffers AM, Wagner A. Neurological complications of cerebral angiography: A retrospective study of complication rate and patient risk factors. Acta Radiol 2000;41:204-10.

4. Schmid UD, Steiger HJ, Huber P. Accuracy of high resolution computed tomography in direct diagnosis of cerebral aneurysms. Neuroradiology $1987 ; 29: 152-9$

5. Anderson GB, Steinke DE, Petruk KC, Ashforth R, Findlay JM. Computed Tomographic Angiography versus Digital Substraction Angiography for the Diagnosis and Early Treatment of Ruptured Intracranial Aneurysms. Neurosurgery 1999;45:1315-22

6. Kato Y, Katada K, Hayakawa M, Nakane M, Ogura Y, Sano K, et al. Can 3DCTA Surpass DSA in Diagnosis of Cerebral Aneurysm? Acta Neurochir (Wien) 2001;143:245-50

7. Matsumoto M, Sato M, Nakano M, Endo Y, Watanabe Y, Sasaki T, et al. Surgery of acutely ruptured cerebral aneurysms aided by three-dimensional computerized tomography angiography without conventional angiography. J
Neurosurg 2001;94:718-27.

8. Pedersen HK, Bakke SJ, Hald JK, Skalpe IO, Anke IM, Sagsveen R, et al CTA in patients with acute subarachnoid haemorrhage. Acta Radiologica 2001;42:43-9.

9. White PM, Teasdale EM, Wardlaw JM, Easton V. Intracranial Aneurysms: CT Angiography and MR Angiography for Detection - Prospective Blinded Comparison in a Large Patient Cohort. Radiology 2001;219:739-49.

10. Young N, Dorsch NWC, Kingston RJ, Markson G, MeMahon J. Intracranial aneurysms: Evaluation in 200 patients with spiral CT angiography. Eur Radiol 2001;11:123-30.

11. Shrier DA, Tanaka H, Namaguchi Y, Konno S, Patel U, Shibata D. CT Angiography in the Evaluation of Acute Stroke. AJNR 1997;18:1011-20.

12. Wildermuth S, Knauth M, Brandt T, Winter R, Sartor K, Hacke W. Role of CT Angiography in Patient Selection for Thrombolytic Therapy in Acute Hemispheric Stroke. Stroke 1998;29:935-8.

13. Harbaugh RE, Schlusselberg DS, Jeffrey R, Hayden S, Cromwell LD, Pluta $\mathrm{D}$, et al. Three-dimensional computed tomographic angiography in the preoperative evaluation of cerebrovascular lesions. Neurosurgery $1995 ; 36: 320-7$

14. Rieger J, Hosten N, Neumann K, Langer R, Molsen P, et al. Initial clinical experience with spiral CT and 3D arterial reconstruction in intracranial aneurysms and arteriovenous malformations. Neuroradiology 1996;38:245-51.

15. Sasiadek M, Hendrich B, Turek T, Kowalewski K, Maksymowicz H. Our own experience with CT angiography in early diagnosis of cerebral vascular malformations. Neurol Neurochir Pol 2001;34:48-55.

16. Fleischmann D, Rubin GD, Bankier AA, Hittmair K. Improved uniformity of aortic enhancement with customized contrast medium injection protocols at CT angiography. Radiology 2000;214:363-71

17. Hartmann I.J, Lo RT, Bakker .J, de Monye W, van Waes PF, Pattynama PM. Optimal scan delay in spiral CT for the diagnosis of acute pulmonary embolism. J Comput Assist Tomogr 2002;26:21-5.

18. Brink JA. Contrast optimisation andscan timing for single and multidetectorrow computed tomography. J Comput Assist Tomogr 2003;27:S3-8.

19. Hittmair K, Fleischmann D. Accuracy of predicting and controlling time-dependent aortic enhancement from a test bolus injection. J Comput Assist Tomogr $2001 ; 25: 287-94$.

20. Rankin SC. Spiral CT: Vascular applications. Eur J Radiol 1998;28:18-29.

21. van Hoe L, Marchal G, Baert AL, Gryspeerdt S, Mertens L. Determination of scan delay time in spiral CT-angiography: Utility of a test bolus injection. J Comput Assist Tomogr 1995;19:216-20.

22. Nakajima Y, Yoshimine T, Yoshida H, Sakashita K, Okamoto M, Kishikawa M, et al. Computerized tomography angiography of ruptured cerebral aneurysms: Factors affecting time to maximum contrast concentration. J Neurosurg 1998;88:663-9

23. Puskas Z, Schuierer G. Kreislaufzeitbestimmung zur Optimierung der Kontrastmittelapplikation bei der CT-Angiographie. Radiologie 1996;36:750-7.

24. Hunt WE, Hess RM. Surgical risk as related to time of intervention in the repair of intracranial aneurysms. J Neurosurg 1968;28:14-20.

25. Fisher CM, Kistler JP, Davis JM. Relation of cerebral vasospasm to subarachnoid hemorrhage visualized by computerized tomographic scanning. Neurosurgery $1980 ; 6: 1-9$.

26. Schad N, Schepke P, Rohde U, Schepke H, Schmid V, Breit A. Timing of exposure in angiographic computed tomography. Cardiovase Intervent Radiol $1981 ; 4: 59-65$

Accepted on 01.11.2004.

\section{Invited Comments}

CT-Angiography is increasingly accepted as the primary tool to search for intracranial aneurysms in patients with SAH. To ensure optimal enhancement of intracranial arteries efficient bolus timing is crucial. Currently there are three possibilities for bolus timing: Using a fixed delay-time (e.g. $20 \mathrm{sec}$ ) will usually lead to good results but in some patients opacification of arteries will not be optimal. The test-bolus method and bolus-tracking techniques both allow for ideal bolus timing and are therefore accepted as the preferred methods when optimized vessel opacification is required. For those who insist on using a fixed delay-time the study of Lukosevicius et al gives important information. The study shows that the ideal delay-time not only depends on the age of a patient with SAH but also on the clinical stage as measured by means of the Hunt-Hess classification. This finding may be explained by the well-known fact that the intracra- 
nial pressure increases in a poor clinical stage. Thus in older patients with a Hunt-Hess grade $>2$ a fixed delay of 20 sec will most likely not lead to sufficient opacification of arteries in most cases. The paper nicely shows that bolus timing should always be individualized especially in patients with

\section{Invited Comments}

Lukosevicius et al have made a clinically useful model by investigating the factors influencing an optimal delay time for computed tomographic angiography (CTA) after subarachnoid hemorrhage, and found that age and neurological status were significant predictive factors.

CTA has become a promising method for neurosurgical procedures. It is less invasive and provides us important information. As for the determination of scan delay, I agree with the authors that dynamic prescan is time-consuming. However, what is important is that this field of examination is not only to improve the statistical model, but also to find out the adequate timing of contrast medium for an individual patient, which requires further study.

I routinely use CTA in managing subarachnoid hemorrhage, mainly for the evaluation of the aneurysm and cerebral vasos-
SAH and a fixed delay cannot be recommended.

Bernd F. Tomandl

Department of Diagnostic and Interventional Radiology, Nuremberg/ Germany. E-mail: bernd_tomandl@hotmail.com

pasm. The method for determining scan delay in our system is called "SureStart" which is the automatic triggering system. When the CT value reached the threshold at the cervical carotid artery, helical scanning was started automatically, ${ }^{[1]}$ which is also one of the promising methods for clinical practice.

Yasunari Otawara

Department of Neurosurgery, Iwate Medical University, Japan. E-mail: ootawara-nsu@umin.ac.jp

\section{Reference}

1. Otawara Y, Ogasawara K, Ogawa A, Sasaki M, Takahashi K. Evaluation of vasospasm after subarachnoid hemorrhage by use of multislice computed tomographic angiography. Neurosurgery 2002;51:939-43. 